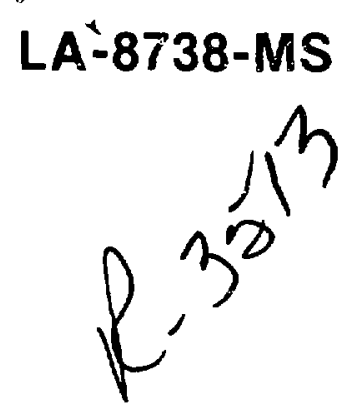

\title{
Pr. 2520
}

\section{A Method of inferring the Magnetic Field Profiles in the Reversed-Field Pinch}

\section{MASTER}

$\frac{0}{5}$ 


\title{
A METHOD OF INFERRING THE MAGNETIC FIELD PROFILES
}

IN THE REVERSED-FIELD PINCH

by

Richard Gerwin

\begin{abstract}
A diagnostic procedure is proposed that displays the toroidal and poloidal magnetic field profiles in the form of power series, through $r^{6}$. In addition to the usual external measurements, only one internal point magnetic measurement is required, if cylindrical symmetry and pressure balance are assumed. (The plasma pressure profile is also assumed to be measured nonintrusively; alternatively, it might be ignored if beta is known to be sufficiently small.) The (zero-beta) method is tested here against full magnetic-profile shot-averaged data obtained from a multiple-probe device in $2 \mathrm{~T}-40$ with a ceramic liner. The agreement is quite good. The procedure seems simple enough for on-line use in RFP experiments. The avallability of such profiles should enhance the effective application of theory to the RFP.
\end{abstract}

\section{INTKODUCTION}

It is important to be able to infer the toroidal and poloidal magnetic field profiles in the Reversed-Field Pinch (RFP) without using large internal multiple-probe instruments that degrade the plasma. In this report, a simple method of infering the field profiles by means of external magnetic measurements, and a single-point internal magnetic measurement is described. The method is tested against profiles obtained with extensive, multi-point internal probe devices, and the agreement seems to be very good. Furthermore, the method appears to be simple enough for on-line use. 
Knowledge of the field profiles in an undegraded RFP plasma has several important consequences for knowledge of:

- Poloidal Flux Loss

* Energy Confinement Time

- Transport Code Physics

- :HD Stability

- Microstability

We shall now briefly dwell on each of these topics.

1) From Faraday's law, we have a relation between the loop voltages on the axis and at the wall, in terms of the poloidal flux, namely

$$
v_{z}(a)=v_{z}(0)+\frac{d}{d t} i 1 j_{0}^{a} B_{G}(r) d r ;
$$

(Hiere, $1=2 \pi R$ is the major circumference.)

Since the gap voltage at $r=a$ is measured, and assuming magnetic fields and hences the polodial flux can be somehow inferred to a satisfactory accuracy, one could then use $\mathrm{Eq}$. (1) to calculate $v_{z}(0)$, the loop vcltage on the magnetic axis. Since knowledge of the magnetic proiiles implies knowledge of the curreni density $j(\mathrm{r})$, ore could then relate $\mathrm{v}_{z}(\mathrm{o})$ to $\mathrm{J}_{z}(\mathrm{o})$ by means of : parallel resististy, $\therefore_{. \prime}$. Thus, one could have an indirect measurement of the parallel resistivity on the magnetic axis. This should then be compared with the resistivity obtained from a measurement of the electron temperature.

2) From power balance, one knows that the input power is partitioned so as to raise the internal magnetic and thermal energies, and also to supply a throughput of energy to the walls iue to uncontrollable losses (thermal conduction, impurity radiation, and plasma radiation). Thus

$$
I_{z} V_{z}+I_{g} V_{g}=\frac{d}{d t}: 1 \int_{0}^{a} \frac{B^{2}}{2 \mu_{0}} 2 \pi r d r j+\frac{d}{d t}\left(1 \int_{0}^{a} \frac{P}{\gamma-1} 2 \pi r d r\right)+\left(1 \int_{0}^{a} \frac{P}{\eta-1} 2 \pi r d r\right) t^{-1},
$$

where $t_{E}$ is an overall effective thermal energy confinement time. 
Now the toroidal and poloidal currents and gap voltages are measured, and assuming that the fiela profiles can be somehow inferred, the magnetic energy could then be estimated. In addition, if the plasma pressure profile could be measured nonintrusively (laser interferometry for the density, for example), then Eq. (2) could provide an estimate of the effective energy confinement time $t_{F}$. (Macroscopic fluctuations could be explicitly modeled into $\mathrm{Eq}$. (2). Otherwise, they will appear as an apparent drain on the plasma in the form of a reduced $t_{F}$ ).

3) Inferred magnetic field profiles (and measured plasma pressure profiles) could be used as initial conditions for, and surve as running comparisons with, cylindrical RFP transport codes. Such tandem modes of investigation could provide valuable clues as to the nature of the effective transport coefficients.

4) Such profiles could also be checked against ideal and resistive MHD stability criteria. (To do a complete job here, it is necessary to have measured the plasma pressure profile.) If the macroscopic fluctuations are sufficiently small, one might expect such mean-field (ensemble- or time-averaged) profiles to be near marginal stability.

5) Such profiles could also be examined for their susceptibility to micru-instabilities of various kinds. (Here it is desirable that the field profiles are known so accurately that drift velocities associated with the deriod current densities are physically meaningful.)

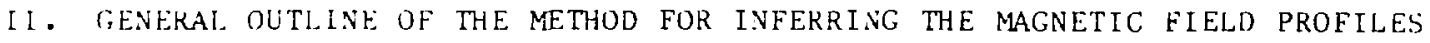

We focus here on RFPs of large aspect ratio and therefore we ignore toroidal effects. (The $Z T-40$ device has $A=5.7$. ) Then, since the toroidal field, $B_{z}(r)$, is an even function with respect to the magnetic axis, an expansion in radial power series through $r^{6}$ involves the determination of four coefficients. (The poloidal field, $B_{\theta}(r)$, can then be determined from pressure balance if the plasma pressure profile is known to sufficient accuracy.)

The use of a power series expansion here is very convenient, as only trivial algebraic manipulations are required to calculate any quantity of interest. The method is also "unbiased". For instance, the leading term of the $B_{z}$ expansion is not taken to be a Bessel function. 
There are three external measurements that are routinely available, namely, the edge values of the toroidal and poloidal magnetic fields, and the iaternal toroidal flux. A single internal point measurement of the magnetic fiold is then required to determine the fourth coefficient of the power series, the coefficient $r^{6}$.

Moreover, we find that this fourth coefficient generally has to be nonzern if the three eaternal measurements are to be satisfied by the power series. Thus, nne internal point measurement appears to be really essential to determine : ise Eourth coefficient.

Such an iatrrnal single-point measurement is most straightforwardy perfortned by i single magnetic probe placed near the wall but it could also be performed nonuestrictivesy. (An example of the lateer would be the nbseration of the zecman effect at a known point in the path of a narrow atomir beim ia ned Lirough the plasma along a minor chord.) Also, the internal point measuremut "ould be replaced by a Faraday rotation measurement, thus sianling an entire interial chord, provided the density profile is independently meisisred.

II1. EXPIICI F FURMIAS FUR THE DETERMT NATLON OF THE COEFFICIENTS

We siball refresint the internal toroidal and poloidal magnetic fields as the respectiof puwer series

$$
\begin{aligned}
& B_{z}=B_{1} 1+a_{-} r^{2}+a_{4} r^{4}+z_{6} r^{b}, \\
& B_{1}=B_{1},{ }_{1} r+\cdot_{3} r^{3}+y_{5} r^{5},
\end{aligned}
$$

where $B_{n}$ is the toridil field on the magnetic axis.

Here, $r$ is normalizud to the discharge tube radius, a, so that $0 \leqslant r \leqslant 1$. The coefficients $z_{2}, z_{4}, z_{6}, \eta_{1}, u_{3}, j_{5}$, and $B_{0}$, are all to be determined from available measurements. Here, we have noted that, in a circularly symetric, cylindrical configuration, the toroidal and poloidal magnetic fields are respectively eien and odd functions across the magnetic axis. The externally measured quantities are $B_{2}$,

$$
\left.B_{2}(1)=B_{0} ; 1+z_{2}+z_{4}+z_{6}\right),
$$


and $\mathrm{B}_{\theta}$,

$$
B_{0}(1)=B_{0}\left(\theta_{1}+\theta_{3}+\theta_{5}\right),
$$

and the toroidal flux in the discharge tube, $\Phi_{z}$,

$$
\bar{B}_{z} \equiv \frac{\Phi z}{\pi a^{2}}=\int_{0}^{1} B_{z} 2 r d r=\int_{0}^{1} B_{L} d x,
$$

with $x \equiv r^{2}$. Hence

$$
\bar{B}_{z}=B_{0}\left(1+\frac{1}{2} z_{2}+\frac{1}{3} z_{4}+\frac{1}{4} z_{6}\right) .
$$

In the following, we shall refer to the traditional $F$ and $\theta$ symbols used tc describe the RFP behavior.

$$
\begin{aligned}
& F \equiv B_{z}(1) / \bar{B}_{z} \\
& 0 \equiv B_{\theta}(1) / \bar{B}_{z}
\end{aligned}
$$

Into the Eq. (8a), $\mathrm{B}_{z}(1)=\overline{F B}_{z}$, we substitute the expressions, Eq. (5), and Eq. (7). The result can be used to solve for $z_{4}$, as follows:

$$
z_{4}=-\frac{1-F+z_{2}(1-F / 2)+z_{6}(1-F / 4)}{1-F / 3} .
$$


Then, we turn back to Eq. (5) itself, substituting the above expression for $z_{4}$, and then solving for the coefficient $z_{2}$. In so doing, we write

$$
\frac{B_{z}(1)}{B_{0}}=\frac{B_{z}(1)}{B_{\theta}(1)} \frac{B_{\theta}(1)}{B_{0}}=\frac{F}{\theta} \frac{1}{u}
$$

wherein we have introduced our basic unknown, $u$, as

$$
\mathrm{u} \equiv \mathrm{B}_{\mathrm{o}} / \mathrm{B}_{\theta}(\mathrm{l})
$$

Thus, $u$ is the central field normalized to the edge value of the poloidal field.

There results the following expression for $z_{2}$.

$$
z_{2}=\frac{\left[(F / O u)-1+(1-F) /(1-F / 3)-z_{6}\{1-(1-F / 4) /(1-F / 3)\}\right]}{[1-(1-F / 2) /(1-F / 3)]} .
$$

Notice that in Eqs. (9) and (12), z6 is so far treated as a parameter. It will eventually be adjusted so that an internal point measurement can be satisfied.

Next, we turn to pressure balance in order to determine the $\theta$ coefficients. Pressure balance may be expressed as follows:

$$
B_{\theta}^{2}=\frac{1}{x_{0}^{\prime}}{ }_{0}^{x}\left(B_{z}^{2}+2 \mu_{0} P\right) d x-\left(B_{z}^{2}+2 \mu_{0} P\right)
$$

where $x \equiv r^{2}$. We shall suppose that the plasma pressure profile can somehow be measured, and shall write it in the following form:

$$
P=P_{0}\left(1+P_{2} r^{2}+P_{4} r^{4}+P_{6} r^{6}\right)
$$


From Eqs. (3) and (4), we note that both fields are taken to have the comon amplitude factor, $B_{0}$. We shall therefore divide Eq. (13) by $B_{0}{ }^{2}$ and write

$$
\frac{2 \mu_{\mathrm{o}} \mathrm{P}_{\mathrm{O}}}{\mathrm{B}_{\mathrm{O}}{ }^{2}}=\frac{2 \mu_{\mathrm{o}} \mathrm{P}_{\mathrm{O}}}{\mathrm{B}_{\mathrm{G}}{ }^{2}(1)} \frac{\mathrm{B}_{\mathrm{g}}{ }^{2}(\mathrm{l})}{\mathrm{B}_{\mathrm{O}}{ }^{2}}=\beta_{\mathrm{P}} / \mathrm{u}^{2},
$$

where we recall Eq. (IL) for $u$, and where we have here defined $B_{p}$, a poloijal beta, with respect to the central plasma pressure, rather than the average plasma pressure.

Then, upon expanding each side of Eq. (13) through $\mathrm{r}^{6}$, and equating the coefficients of like powers of $r$, we find the following relations among the coefficients.

$$
\begin{aligned}
& \theta_{1}=\sqrt{-(1 / 2)\left(2 z_{2}+\beta_{\mathrm{P}} \mathrm{P}_{2} / u^{2}\right)} \\
& \theta_{3}=-\left(z_{2}^{2}+2 z_{4}+\beta_{P^{P}} P_{4} / u^{2}\right) /\left(3 \theta_{1}\right) \\
& \theta_{5}=\left[-(3 / 4)\left(2 z_{2} z_{4}+2 z_{6}+\beta_{P} P_{6} / u^{2}\right)-\theta_{3}^{2}\right] /\left[2 \theta_{1}\right]
\end{aligned}
$$

It makes no sense to try to use the pressure balance relation more accurately than indicated here (i.e., to higher order in $r$ ), because the higher coefficients that would then appear are inaccessible within the context of only one internal point measurement.

Ideally, one would like to have available a nondestructive measurement of the plasma pressure profile $\left(B_{p}, P_{2}, P_{4}, P_{6}\right)$ to use in Eqs. $(16)$. There are such methods for measuring density and electron temperature profiles, but the ion temperature profile measurement presents a difficult problem.

To summarize, $z_{2}$ is known in terms of $u$ from Eq. (12), which also contains the external measurements, $F$ and $\theta$. The coefficient $z_{+}$is known in terms of $z_{2}$ from $\mathrm{Eq}$. (9), which also contains $F$. The $\theta$ coefficients are known in terms of $z_{2}$ and $z_{4}$ from Eqs. (16), which also contain u explicitly through the plasma pressure profile coefficients. In all of the above, the coefficient $z_{6}$ is retained as an adjustable parameter. The reasons for this will become clear in the following section. 
The final step in actually finding the coefficients of our power series is to substitute the expressions for the $\theta$ coefficients (Eq. (16)) into Fq. (6). This equation can obviously be written

$$
u=1 /\left(\theta_{1}+\theta_{3}+\theta_{5}\right),
$$

and it amounts to a single nonlinear equation for $u$, with $z_{6}$ as a parameter. Having obcained a solution, $u$, of $\mathrm{Eq} \cdot(17)$, the coefficients $z_{2}, z_{4}, G_{1}$, $u_{3}, u_{5}$, may be explicitly calculated as discussed above, for each trial value of $z_{6}$. They then yield magnetic field profiles which satisiy all of the external measurements. However, the coefflcient $z_{6}$ is still free, and nust be Fixed by an internal point measurement of some sort.

\section{TV. COMPARISON WITH EXPERIMENT}

A code has been written to solve Eq. (17) and to display the resulting field profiles. In this section the procedure will be tested by comparing the results with full (minor radius) profiles obtained from multi-probe meisurements on the Los Alamos Reversed-Field Pinch, ZT-40. Since the pressure profile has not yet been measured, and since beta is believed to be small (from the available temperature measurements and reasonable estimates of pumpout), we shall set the plasma pressure to zero when making this comparison. Note that the shots were obtained on the ceramic liner version of 7t-40. No quiescent periods were observed when the multi-probe device was inserted. Consequently, in order to obtain cylindrical mannetic profiles, it was necessary to average over fluctuations in some way.

In these nominally identical shots, the ten pairs of probe measurements, two centimeters apart, were made $335 \mu s$ after the start of the toroidal current, and then were averaged over the shots. The shot numbers are 3856 , $3857,3858,3861,3862$, and 3863. The measured values of $F$ and $\Theta$ were $F=$ -0.18 and $O=1.70$. This value of $\theta$ corresponds to an edge value of $B_{\theta}$ consistent with the total coroidal current, and constitutes a correction that had to be separately inserted due to the influence of the diagnostic port and toroidal effects on the $B_{\theta}$ probes near the edge. Jim Phillips also applied $B_{\theta}$ corrections that insure that the poloidal flux is properly balanced with 
respect to the magnetic axis. These corrections (to remove the influence of the diagnostic port) are shown as the dashed curve In Fig. 1. By coincidence, they produce an edge value of $B_{\theta}$ consistant with the total toroidal current. (What this implies is that the decrease of the outer $B_{G}$ due to the toroidal $\mathrm{R}^{-1}$ effect must be approximately balanced by the increase in the outer $B_{\partial}$ due to the toroidal shift. One would not expect this coincidence to routinely occur.) In general, to use the methods presented here for cylindrical geometry, one should take the "measured" $B_{\theta}(1)$ value to be defined by the total toroidal current, $I$, as we do now. (Of course, I is now shot-averaged at $335 \mu \mathrm{s.}$ )

In Figs. 2 and 3 we now plot the two sides of Eq. (17), u $=F(u)$, with $z_{6}$ as a parameter, for the above experimental values of $F$ and $\theta$, with plasma pressure ignored $\left(\beta_{P}=0\right)$. Notice that there are no solutions for $z_{6}=0$. In other words, a power series for $B_{z}$ that is terminated at $r^{4}$ cannot satisfy the oxterilal constraints on $F$ and $\Theta$ for vanishing (or small) plasma pressure. Thus, a fourth measurement becomes mandatory in order to fix the value of the coefficient $z_{6}$. Note also, that for some values of this coefficient, there is more than one solution to Eq. (17); that is, distinct magnetic profiles can satisty one set of external measurements.

In making further comarison with experiments, we shall pretend that we experimentally know $B_{z}$ either at the probe positior, $r=16 \mathrm{~cm}$, or at $\mathbf{r}=$ $18 \mathrm{~cm}$. (The tube radius is $\mathrm{r}=20 \mathrm{~cm}$.) Experimentally, one has

$$
\begin{aligned}
& \mathrm{B}_{z}(16) / \mathrm{B}_{\theta}(20)=0.289, \\
& \mathrm{~B}_{z}(18) / \mathrm{B}_{\theta}(20)=0.084 .
\end{aligned}
$$

From Figs. 2 and 3 , we see that, for $z_{6}=-0.3$ and -0.5 , there is only one root, whereas for $z_{6}=-0.4$ there are three roots to consider. The results nay be tabulated as follows: 


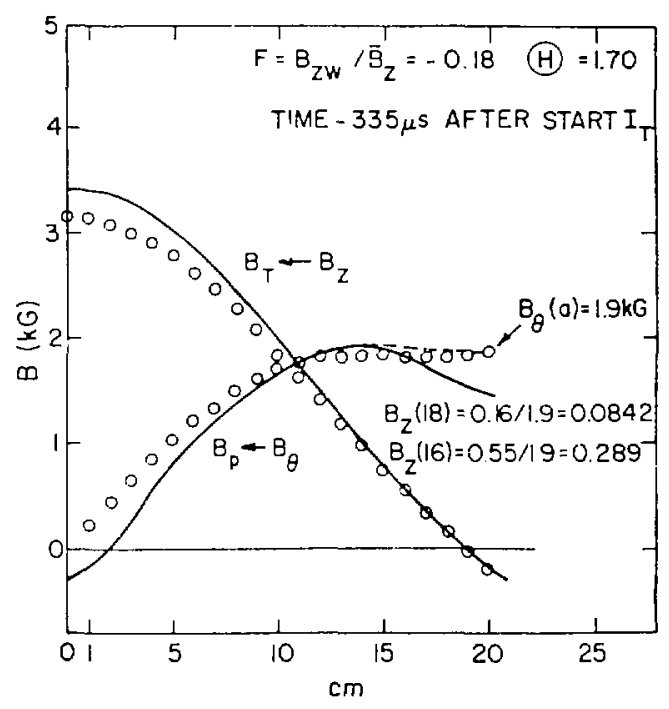

Fig. 1. Comparison of $\mathrm{ZT}-40$ shotaveraged multi-probe data (solid curves drawn through probe data at $r=0,2,4, \cdot \cdot, 20 \mathrm{~cm}$ ) with the power series inference (open circles). The shot-averaged data were obtained from J. Phillips, CTR-DO. The multi-probes were constructed by $L$. Burkhardt, CTR-2. The dashed curve is the corrected $B_{\theta}$ profile.

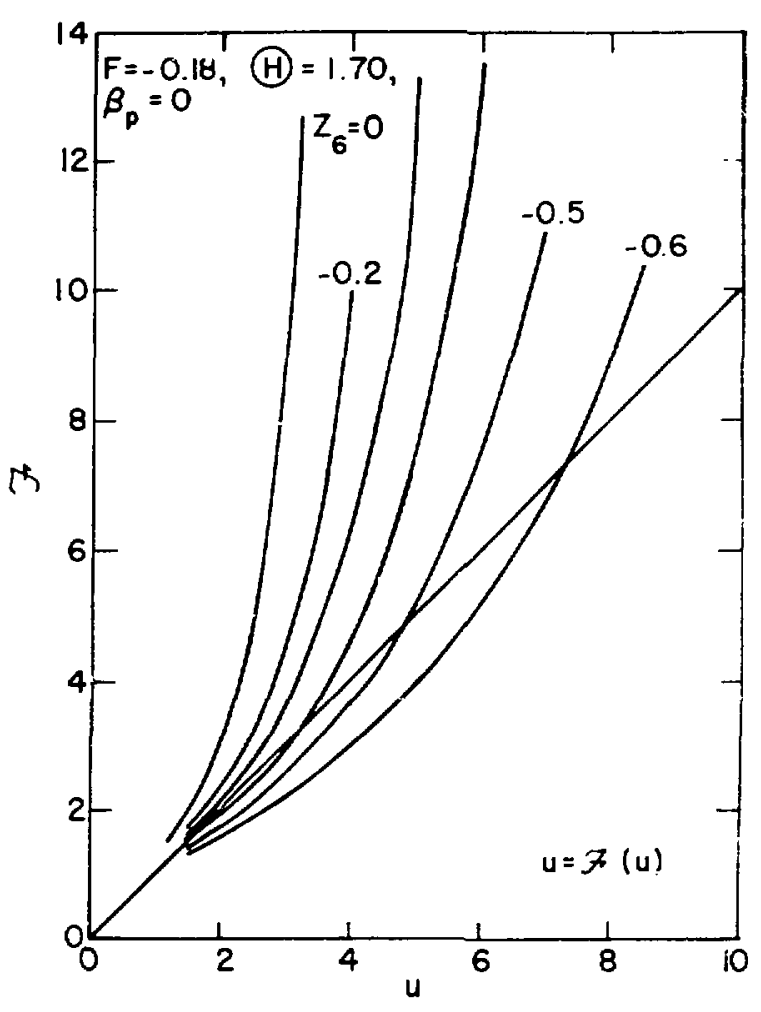

Fig. 2. A plot of Eq. (17) for various values of the coefficient $z_{6}=$

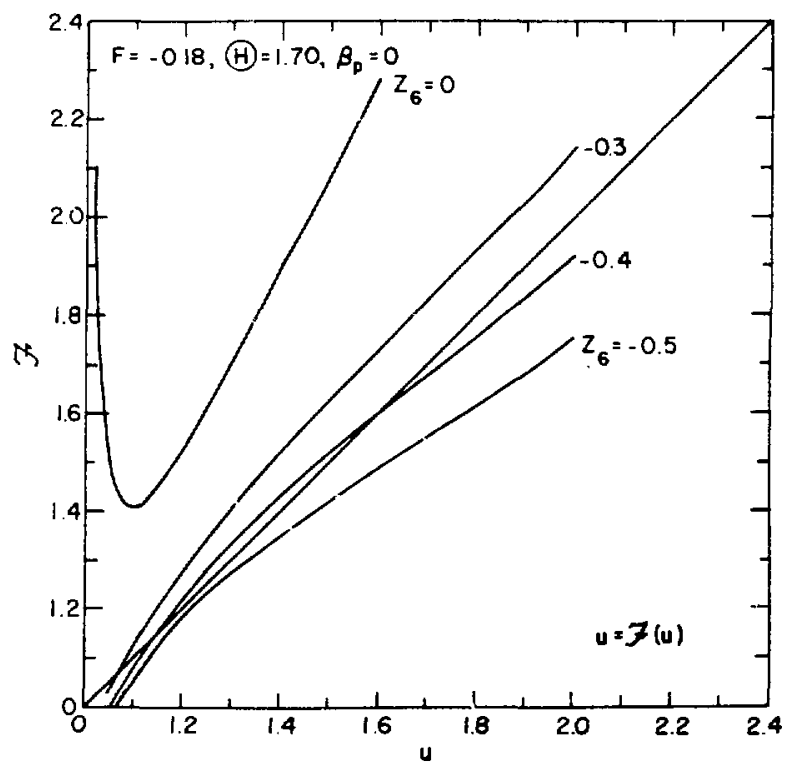

Fig. 3.

A plot of Eq. (I7), as in Fig. 2, but examined in detail at small values of u. 
TABLE I

A Scan of Roots Corresponding to the Measured $(F, \odot)$ !alues

$\begin{array}{cccr}Z_{6} & u & B_{z}(16) & B_{z}(18) \\ -0.5 & 4.775 & -0.686 & -0.677 \\ -0.4 & 3.095 & -0.167 & -0.276 \\ & 1.579 & 0.316 & 0.107 \\ -0.3 & 1.141 & 0.456 & 0.218 \\ & 1.079 & 0.472 & 0.229\end{array}$

All of these roots satisfy the external measurements, $F$ and 0 .

From TABLE I, we see that the root that comes the closest to satisfying either of the internal measurements, Eq. (18), is the middle root of the case $z_{6}=-0.4$. Therefore, we investigate this middle root more closely in TABILE II.

TABLE II

A Scan of the :iiddle Root

$\begin{array}{cccc}Z_{6} & u & B_{z}(16) & B_{z}(18) \\ -0.41 & 1.524 & 0.334 & 0.122 \\ -0.39 & 1.643 & 0.295 & 0.090 \\ -0.387 & 1.665 & 0.288 & 0.085 \\ -0.386 & 1.673 & 0.286 & 0.083 \\ -0.385 & 1.680 & 0.283 & 0.0806 \\ -0.380 & 1.722 & 0.269 & 0.070\end{array}$

From TABLE II, we see that the value, $z_{6}=-0.387$, yields a root which not only satisfies the measured $(F, O)$ values, but also comes to within three decimal places of both $B_{z}(16)$ and $B_{z}(18)$. (This happy circumstance must be partly fortuitous, since the measurements are not all that accurate, and since an average over several shots has been performed.) The corresponding profiles have been plotted as the open circles in Fig. 1.

Except for a small toroidal shift, inaccessible to our model, the agreement is seen to be quite good. Moreover, the root finding and scanning procedure is simple enough to be used on-line. 


\section{CONCLUSION}

In conclusion, we have seen that an RFP magnetic profile inference can be made in terms of power series through $r^{6}$, provided that a single internal point magnetic measurement is used to supplement the external measurements. The best near-term approach would be to place a dogleg probe near the plasma edge, along the wall away from the diagnostic port, or a straight probe in conjunction with a metal flap that covers the port. In the long term, a nonintrusive Faraday rotation or cyclotron radiation measurement to infer $B_{z}$ at $\mathbf{r}=0$ would be preferable, since the polynomial would then be "tied down" at both ends. Finally, the procedure described herein can only realize its full value in conjunction with a nonintrusive plasma pressure profile meisurement. 